\title{
Remission of an Intracardiac Melanoma Metastasis after Tremelimumab Therapy
}

\author{
Sebastian Spethmann ${ }^{a, b} \quad$ Uwe Trefzer $^{c} \quad$ Fabian Knebel $^{a}$ \\ ${ }^{a}$ Medizinische Klinik für Kardiologie und Angiologie, Campus Mitte, Charité - \\ Universitätsmedizin, ${ }^{\mathrm{b}}$ Bundeswehrkrankenhaus Berlin, Abteilung I - Innere Medizin, and \\ 'Dermatologikum Berlin, Berlin, Germany
}

\section{Key Words}

Tremelimumab · Intracardiac melanoma metastasis · Tumour regression

\begin{abstract}
Tremelimumab is a fully human monoclonal antibody specific for human cytotoxic $T$ lymphocyte-associated antigen 4. Administration of tremelimumab to patients with locally advanced and metastatic melanoma has resulted in a subset of patients with durable objective tumour regressions. Our echocardiographic images impressively show the effects of this new antibody in a patient with cardiac metastatic malignant melanoma.
\end{abstract}

(c) 2014 S. Karger AG, Basel

A 51-year-old woman with stage IV melanoma was enrolled in a tremelimumab study [1]. She was initially diagnosed with malignant melanoma on her back in May 2009. On October 27, 2010, prior to her enrolment in the study, she underwent a transthoracic echocardiography examination. The examination revealed a massive tumour metastasis, probably haematogenic spread through the pulmonary veins, filling out almost the whole left atrium (initial size: fig. 1a, fig. 2a). In November 2010, a therapy with tremelimumab 15 $\mathrm{mg} / \mathrm{kg}$ once every 90 days was initiated. Both the patient subjectively and the subcutaneous metastasis responded favourably to the therapy. A follow-up echocardiography 4 weeks later showed regression of the intracardiac metastasis (fig. 2b). Moreover, 6 weeks later, the left atrial tumour had completely disappeared (fig. 1b, fig. 2c). Unfortunately, 2 months later, the patient died at home. 
Spethmann et al.: Remission of an Intracardiac Melanoma Metastasis after Tremelimumab Therapy

\section{Reference}

1 Ribas A, Kefford R, Marshall MA, Punt CJ, Haanen JB, Marmol M, et al: Phase III randomized clinical trial comparing tremelimumab with standard-of-care chemotherapy in patients with advanced melanoma. J Clin Oncol 2013;31:616-622.
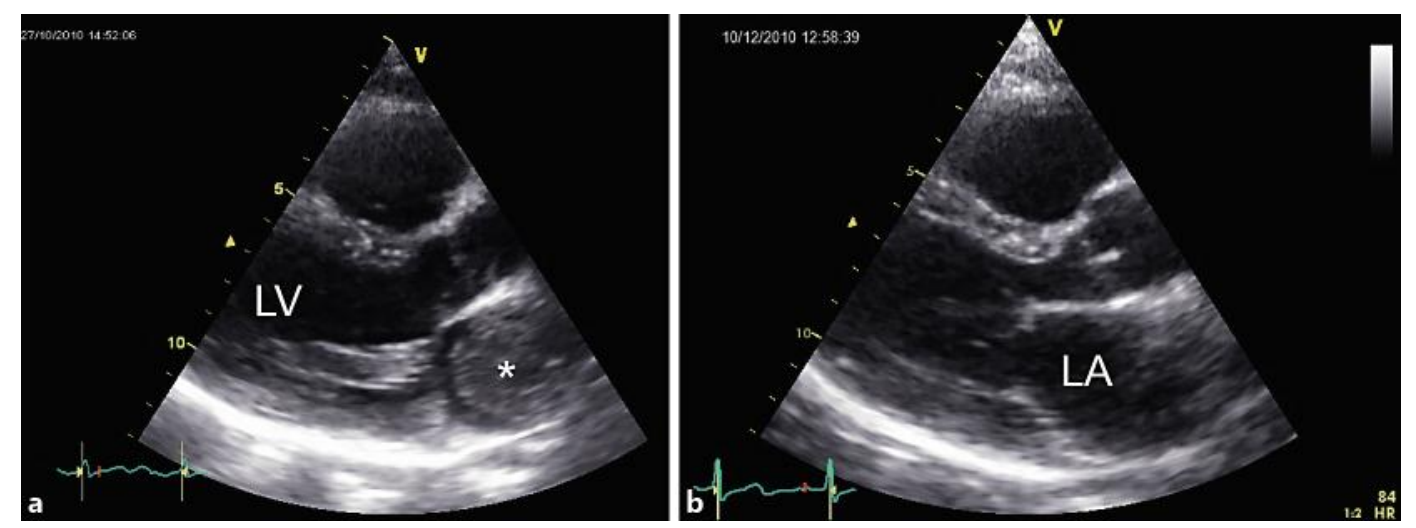

Fig. 1. The parasternal long axis view shows a sessile tumour $\left({ }^{*}\right)$ filling out almost the whole left atrium (LA) (a) prior to tremelimumab therapy. Three months later, the atrial tumour had completely disappeared (b). LV = Left ventricle. 
Spethmann et al:: Remission of an Intracardiac Melanoma Metastasis after Tremelimumab Therapy
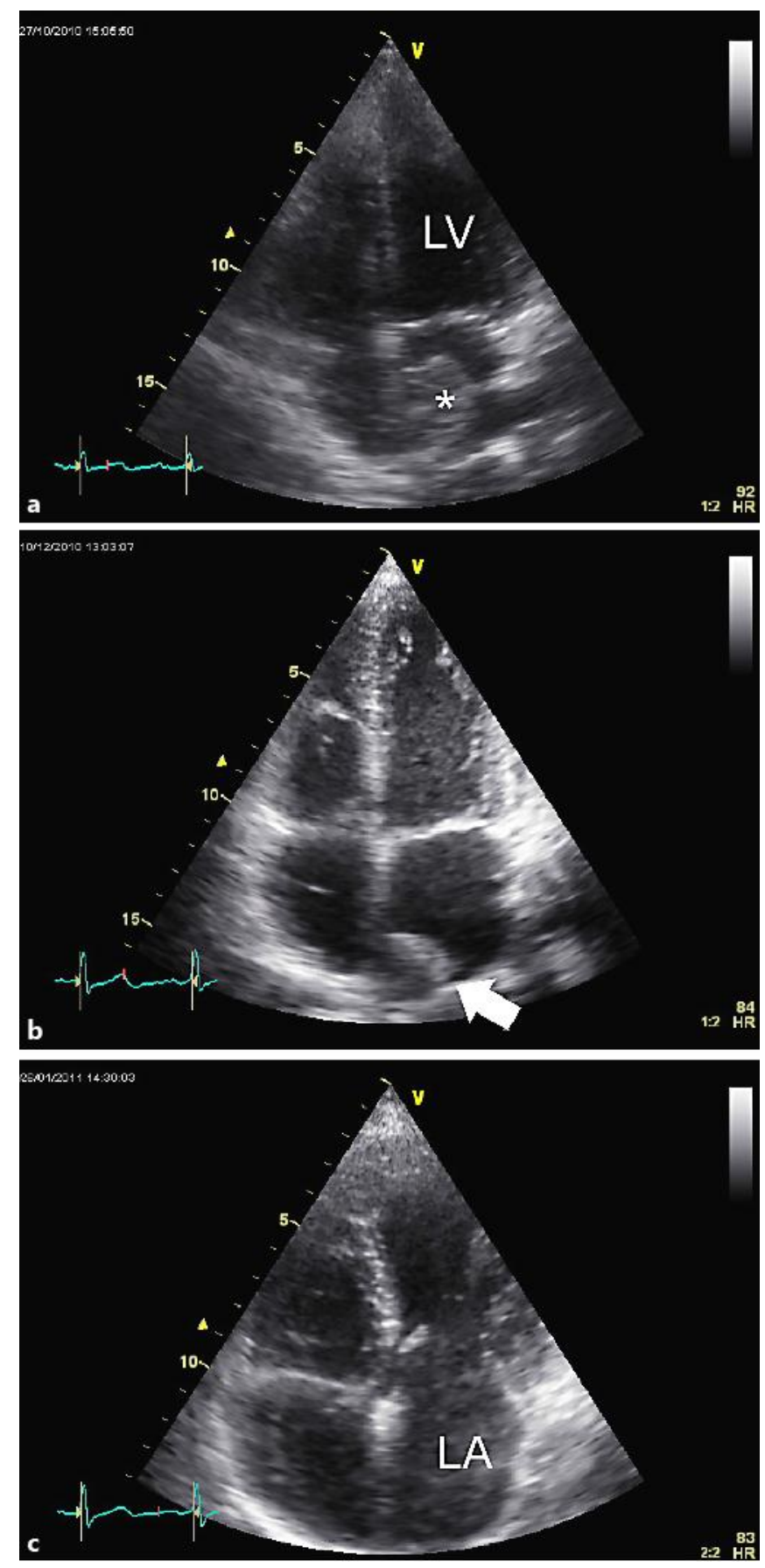

Fig. 2. a The four-chamber view reveals a massive left atrial tumour $\left({ }^{*}\right)$, probably out of pulmonary veins. b Thirty-six days after the first tremelimumab infusion regression of the intracardiac metastasis occurred (arrow). c Six weeks later, the left atrial tumour had completely disappeared. $\mathrm{LA}=\mathrm{Left}$ atrium; $\mathrm{LV}=\mathrm{left}$ ventricle. 